\title{
THE MAGELLANIC CLOUDS: DISTANCE, STRUCTURE, CHEMICAL COMPOSITION
}

\author{
M.W. FEAST \\ South African Astronomical Observatory \\ PO Box 9, Observatory 7935, Cape \\ South Africa
}

ABSTRACT. As an introduction to the symposium the related problems of the distances, structures and chemical compositions of the Magellanic Clouds are reviewed.

\section{Introduction}

As an introduction to the detailed papers which follow it may be useful to make some general comments on the related problems of the distance, structure and chemical make-up of the two Clouds.

The part of the IAU Symposium 20 (Kerr \& Rodgers 1964) which dealt with the Magellanic Clouds was held here in Sydney 27 years ago. The proceedings of that meeting contain hardly any discussion of the distances of the Clouds. This may at first seem strange. After all, long before that - at the 1952 Rome IAU General Assembly - Thackeray had announced the discovery by himself and Wesselink of RR Lyraes in Cloud clusters. These were 1.5 mag fainter than expected, and together with Baade's failure to detect RR Lyraes in M31 and NGC 205, announced simultaneously, were the basis for a doubling of the extragalactic distance scale. The problem in 1963 was both the difficulty in obtaining good apparent magitudes for RR Lyraes in the Clouds, a problem only recently solved by Alistair Walker and others, and the uncertainty in the precise absolute magnitude of RR Lyraes.

It is worth recalling that the discovery of the Cloud RR Lyraes was, in addition, important in establishing that the Clouds must be treated as full-blown galaxies, containing the different types of objects that make up a galaxy. The view held by Baade (1951) and others at that time was that the LMC was a pure population I system with no very old component. Baade's view was based largely on Thackeray's earlier discovery that the globular-like cluster, NGC 1866, in the LMC was actually young, as well as on the supposed absence of RR Lyrae variables in the Clouds.

\section{Distance moduli}

Cepheid distances to the Clouds were hardly mentioned in Sydney in 1963. There was in fact much concern at the time about the reliability of Cepheids as distance indicators. The available photographic photometry indicated different slopes for the period-luminosity relations in the two Clouds and no-one knew how to handle this. A major step forward was taken by Gascoigne at the 1963 meeting when he presented photoelectric results suggesting that the problem lay in systematic errors in the photographic photometry. 
At the present time Cepheids are probably the most reliable indicators of the distances of the Clouds. Feast and Walker (1987) found moduli of 18.47 (LMC) and 18.78 (SMC) using primarily PL and PLC relations (Martin et al. 1979, Caldwell \& Coulson 1986) based on BVI photometry calibrated using cepheids in open clusters in our galaxy. The cluster distances are based on trigonometrical parallaxes of nearby stars via the Pleiades main sequence. Other recent estimates are close to these Cloud moduli. Laney (cf. Feast 1988) derived 18.52 (LMC) and 18.84 (SMC) from JHK data (Laney \& Stobie 1986a, b, Welch et al. 1987). Visvanathan $(1989,1 \mu \mathrm{m}$ photometry) gives 18.42 (LMC) and 18.83 (SMC). The inter-agreement of the results at various wavelengths suggests the method is sound. The main uncertainty is the basis calibration of the zero point (all the above workers used essentially the same set of Cepheids in galactic clusters with the same set of distances). There is probably an uncertainty of $\sim 0.08$ in the Pleiades modulus alone and Feast and Walker estimate the total uncertainty of the Cloud moduli to be $\sim 0.15$ mag.

Main-sequence fitting of young MC clusters is an alternative way of determining distance moduli. Early work gave a rather low LMC modulus ( 18.1). A rediscussion (VandenBerg \& Poll 1989), matching NGC 2162 (LMC) to a theoretical zero-age main sequence, normalized to the sun and fitted to the Pleiades morphology and adopting $[\mathrm{Fe} / \mathrm{H}]=-0.23$ and $\mathrm{Y}=0.25$, gives a modulus of $\sim 18.4$, in good agreement with the Cepheids. The result is sensitive to the adopted metallicity. A decrease of $[\mathrm{Fe} / \mathrm{H}]$ by 0.1 results in a decrease in the modulus by $\sim 0.1$. The adopted metallicity is close to the likely LMC value (cf. Feast 1989a).

The present uncertainty in the absolute magnitudes of the RR Lyrae variables (e.g. Sandage \& Cacciari 1990) makes them less attractive as distance indicators. However the results (cf. summary in Feast 1988 and those to be published) do not differ significantly from the Cepheid moduli. $\mathrm{H} \beta$ photometry of B stars (Shobbrook \& Visvanathan 1987) gives 18.4 (LMC) agreeing with the Cepheids but with a larger uncertainty $( \pm 0.23)$. Mira variables which in the LMC show good PL and PLC relations (Feast $e$ t al. 1989) are important in fixing a consistent relative scale of distances (LMC-SMC-galactic-centre-globular clusters) but their absolute calibration independent of other variables is not accurately determined. Eastman and Kirshner (1989) used a model atmosphere approach to determine an expansion parallax for SN 1987A. They obtain an LMC modulus of $18.45 \pm 0.28$ again agreeing with the Cepheids.

\section{Structure}

The de Vaucouleurs $(1954,1957)$ model of the LMC as a flattened disc tilted to the plane of the sky remains the best overall description. Following early attempts by de Vaucouleurs, Gascoigne \& Shobbrook (1978) used Cepheids to show that the eastern edge of the LMC was nearer than the west. The tilt of the LMC to the plane of the sky may be near $40^{\circ}$ (cf. Westerlund 1990 and Caldwell \& Laney 1990). Data on the thickness of the LMC disc have recently been summarized by Feast (1989b). From its velocity dispersion we infer that the scale height of the young population is small, perhaps in the range 100 to $300 \mathrm{pc}$. However the depth structure may be complex since HI work (Meaburn et al. 1987, Kerr 1989) suggests sheets of gas at different depths through the LMC. As one goes to older populations, the velocity dispersion increases, indicating an increase in scale height. The scatter about the PLC relation for oxygen-rich Mira variables places an upper limit of $\sim 2 \mathrm{kpc}$ on the scale height of this old population. If all the spread in the apparent magnitudes of RR Lyrae variables in the field of NGC 2257 (LMC) is due to a distance spread then this is $\sim 9 \mathrm{kpc}$ and the RR Lyraes could form a spherical sub-system (Walker 1989). However, it is quite possihle that some of this apparent spread is due to a range of absolute magnitudes. 
Caldwell and Coulson (1986) analysed extensive BVI observations of SMC Cepheids and found the system to be an elongated structure seen nearly end on. Infrared observations of SMC Cepheids (Laney \& Stobie 1986a) confirm this picture. At about the same time Mathewson et al. (1986) also published a discussion of the distribution of SMC Cepheids. Their revised results (Mathewson et al. (1988) have been compared with the Caldwell/Coulson model by Feast (1989b). The main result was that when one takes into account the expected uncertainties in the distances derived by Mathewson et al., there is no significant disagreement with Caldwell/Couson. The entire SMC Cepheid system stretches 15 to $20 \mathrm{kpc}$ in the line of sight, though the depth in any one direction is much less than this. Welch et al. (1987) combined their own JHK observations of SMC Cepheids with those of Laney and Stobie (1986b) and analysed them on a simple model in which the depth distribution was assumed to be Gaussian. They found values of $\sigma$ (the true dispersion) between 0.12 and 0.18 mags. To enclose most of the population (on their Gaussian hypothesis) one must take a $\pm 2 \sigma$ scatter on either side of the mean. This gives depth estimates of 13 to $19 \mathrm{kpc}$. Considering the approximate nature of the Gaussian model this agrees remarkably well with the Caldwell/Coulson estimates of 15 to $20 \mathrm{kpc}$. These estimates are close to estimates of the tidal diameter of the SMC and it is not possible to be certain at present whether the SMC exceeds its tidal diameter or not. Martin et al. (1989) have made estimates of the mean distances of different groups of supergiants in the SMC selected according to their kinematics. They conclude that most young objects lie within a depth of less than $10 \mathrm{kpc}$. This result is not contrary to the Caldwell/Coulson model. Further data on the depth structure of the Clouds is given by Caldwell and Laney (1990).

Recently Hatzidimitriou and Hawkins (1989) have published a particularly interesting paper in which they discuss the depth distribution of an old population in the SMC using deep cm diagrams for the outskirts of the Cloud. In the SW the cm diagram shows a circular horizontal branch/giant clump but in the NE they find this clump to be elongated which, they suggest, indicates a great depth in that direction (average depth $17 \mathrm{kpc}$, maximum extent $23 \mathrm{kpc}$ ). Future work will no doubt deal with a possible sensitivity of this result to the corrections applied for foreground/background contamination which produces a strong near vertical column of stars at the colour of the clump (cf. Irwin et al. 1990).

\section{The chemical compositions of the Clouds}

Evidence relating to the chemical abundance of objects of known age in the Clouds was reviewed by Feast (1989a). To this may be added data of F supergiants (Russell \& Bessell 1989, Spite et al. 1989). Leaving aside NGC 330 and NGC 1818 (see below) one finds [Fe/H] -0.2 (LMC) and $\sim-0.5$ (SMC). The metal abundance may fall roughly exponentially with time as one goes to older objects, but the evidence is quite uncertain. At least there are some old clusters with low metallicities ( - 2.0 dex). Much of the apparent spread in metallicity at a given age in either Cloud may be observational. Amongst old objects the three globular clusters in the LMC with accurate RR Lyrae data (Walker 1985, 1989, Walker \& Mack 1988) have a range in metallicities of 0.5 dex which seems real. The O-rich Mira variables may prove useful indicators of chemical composition in old populations, at least in the period range 200 to 400 days. Evidence from the Clouds, the solar neighbourhood, the galactic bulge and the galactic globular clusters suggests that infrared period-colour and period-luminosity relations are closely similar in these various environments (cf. Whitelock et al. 1990). At the same time evolutionary and pulsational arguments as well as Miras in galactic globular clusters indicate that both mass and metallicity increase with period. This suggests that either the P-C and P-L relations are insensitive to metallicity or that the mass- 
metallicity relation is the same everywhere for O-Miras. The latter is only easily understood if all O-Miras are very old. Then the mass-metallicity relation merely reflects the fact that the more metal-rich stars stay longer, at a given mass, on the main sequence (VandenBerg \& Laskarides 1987). Judging by O-rich Miras in galactic globular clusters and the galactic bulge, the range in metallicities amongst O-Miras of the LMC might, in this case, be as high as 1 dex.

The best estimates of the spread of metallicities amongst young objects probably come from HII region data. Pagel et al. (1978) find a dispersion in their abundances in each Cloud of 0.09 dex. All of this could be observational. Five cool AGB stars in the SMC show s-process element enhancement but $[\mathrm{Fe} / \mathrm{H}] \sim-0.5$ similar to other young SMC stars (Smith \& Lambert 1989). Russell and Bessell (1989), from eight F-type supergiants in each Cloud, tentatively suggest a possible (real) dispersion in each Cloud of $\sim 0.2$ dex. However, it has been suggested (Feast 1985) that $\sigma$ is less than 0.15 dex for SMC Cepheids.

This view of a small abundance spread for young objects in each Cloud has recently been challenged by work which attempts to extend abundance determinations to cool ( $\mathrm{T} \sim 4000^{\circ}$ or less) and hot $(\mathrm{OB})$ stars in both Clouds. In particular $[\mathrm{Fe} / \mathrm{H}]$ values of -1.4 and -0.9 have been derived for the stars in the young clusters NGC 330 (SMC) and NGC 1818 (LMC) respectively (Spite $e t$ al. 1986, Richtler et al. 1989, Reitermann et al. 1990). These are about 0.8 lower than for young field stars. It seems unlikely that these abundances can apply to all young or intermediate age clusters in the Clouds. For instance, NGC 2162 in the LMC (see section 2) would have a distance modulus of only $\sim 17.7$ if $[\mathrm{Fe} / \mathrm{H}] \sim-0.9$. A similar result applies to NGC 1866 (LMC) (cf. Chiosi et al. 1989, Brocato et al. 1989) who find that near solar metallicity fits the cm diagram. In addition, in the latter case the colours of Cepheid members indicate $[\mathrm{Fe} / \mathrm{H}] \sim-0.11$ (Feast 1989a) and Washington photometry gives $-0.4 \pm 0.1$ (Schommer \& Geisler 1988), all of which suggests that NGC 1866 has an $[\mathrm{Fe} / \mathrm{H}]$ similar to young field stars $(\sim-0.2)$ and not as low as $\sim-0.9$. The possibility of a large range of metallicities in young clusters, but not in the field, of each Cloud is just one of the many questions we shall be discussing during the next few days.

\section{References}

Baade, W. (1951), Pub. Obs. Univ. Michigan 10, 7.

Brocato, E., Buonanno, R., Castellani, V., Walker, A.R. (1989), Astrophys. J. Suppl. 71, 25.

Caldwell, J.A.R., Coulson, I.M. (1986), M.N.R.A.S. 218, 223.

Caldwell, J.A.R., Laney, C.D. (1990), these proceedings.

Chiosi, C., Bertelli, G., Meylan, G., Ortolani, S. (1989), Ast. Astrophys. 219, 167.

de Vaucouleurs, G. (1954), Observatory 74, 23.

de Vaucouleurs, G. (1957), Ast. J. 62, 69.

Eastman, R.G., Kirshner, R.P. (1989), Ap. J. 347, 771.

Feast, M.W. (1985), Cepheids: Theory and Observations, B.F. Madore (ed.) (Cambridge Univ. Press) p. 157.

Feast, M.W. (1988), The Extragalactic Distance Scale, S. van den Bergh, C.J. Pritchet (eds.) (Ast. Soc. Pacific: San Francisco) p.9.

Feast, M.W. (1989a), The World of Galaxies, H.G. Corwin, L. Bottinelli (eds.) (SpringerVerlag) p.118.

Feast, M.W. (1989b), Recent Developments in Magellanic Cloud Research, K.S. de Boer, F. Spite, G. Stasinska (eds.) (Observatoire de Paris: Paris) p.75.

Feast, M.W., Glass, I.S., Whitelock, P.A., Catchpole, R.M. (1989), M.N.R.A.S. 241, 375.

Feast, M.W., Walker, A.R. (1987), Ann. Rev. Ast. Astrophys. 25, 345. 
Gascoigne, S.C.B., Shobbrook, R.R. (1978), Proc. ast. Soc. Aust. 3, 285.

Hatzidimitriou, D., Hawkins, M.R.S. (1989), M.N.R.A.S. 241, 667.

Irwin, M.J., Demers, S., Kunkel, W.E. (1990), Ast. J. 99, 191.

Kerr, F.J. (1989), The World of Galaxies, H.G. Corwin, L. Bottinelli (eds.) (Springer-Verlag) p. 160.

Kerr, F.J., Rodgers, A.W. (eds.) (1964), IAU Symp 20 The Galaxy and the Magellanic Clouds, (Aust. Acad. Sci: Canberra).

Laney, C.D., Stobie, R.S. (1986a), M.N.R.A.S. 222, 449.

Laney, C.D., Stobie, R.S. (1986b), S.A.A.O. Circulars 10, 51.

Martin, W.L., Warren, P.R., Feast, M.W. (1979), M.N.R.A.S. 188, 139.

Martin, N., Maurice, E., Lequeux, J. (1989), Ast. Astrophys. 215, 219.

Mathewson, D.S., Ford, V.L., Visvanathan, N. (1986), Astrophys. J. 301, 664.

Mathewson, D.S., Ford, V.L., Visvanathan, N. (1988), Astrophys. J. 333, 617.

Meaburn, J., Marston, A.P., McGee, R.X., Newton, L.M. (1987), M.N.R.A.S. 225, 591.

Pagel, B.E.J., Edmunds, M.G., Fosbury, R.A.E., Webster, B.L. (1978), M.N.R.A.S. 184, 569.

Reitermann, A., Baschek, B., Stahl, O., Wolf, B. (1990), Ast. Astrophys. in press.

Richtler, T., Spite, M., Spite, F. (1989), Ast. Astrophys. 225, 351.

Russell, S.C., Bessell, M.S. (1989), Astrophys. J. Suppl. 70, 865.

Sandage, A.R., Cacciari, C. (1990), Astrophys. J. 350, 645.

Schommer, R.A., Geisler, D. (1988), Globular Cluster Systems in Galaxies, J.E. Grindlay, A.G. Davis Philip (eds.) (Kluwer: Dordrecht) p.577.

Shobbrook R.R., Visvanathan, N. (1987), M.N.R.A.S. 225, 947.

Smith, V.V., Lambert, D.L. (1989), Astrophys. J. 345, L.75.

Spite, F., Spite, M., Francois, P. (1989), Ast. Astrophys. $210,25$.

Spite, M., Cayrel, R., Francois, P., Richtler, T., Spite, F. (1986), Ast. Astrophys. 168, 197.

VandenBerg, D.A., Laskarides, P.G. (1987), Astrophys. J. Suppl. 64, 103.

VandenBerg, D.A., Poll, H.E. (1989), Ast. J. 98, 1451.

Visvanathan, N. (1989), Astrophys. J. 346, 629.

Walker, A.R. (1985), M.N.R.A.S. 212, 343.

Walker, A.R. (1989), Ast. J. 98, 2086.

Walker, A.R. (1990), Preprint.

Walker, A.R., Mack, P. (1988), Ast. J. 96, 1362.

Westerlund, B.E. (1990), Ast. Astrophys. Reviews in press.

Welch, D.L., McLaren, R.A., Madore, B.F., McAlary, C.W. (1987), Astrophys. J. 321, 162.

Whitelock, P.A., Feast, M.W., Catchpole, R.M. (1990), Preprint. 